\title{
Maternal genes required for the anterior localization of bicoid activity in the embryo of Drosophila
}

\author{
Hans Georg Frohnhöfer and Christiane Nüsslein-Volhard \\ Max-Planck-Instıtut für Entwicklungsbiologie, 7400 Tübingen, FRG
}

\begin{abstract}
The maternal gene bicoid $(b c d)$ organizes anterior development by means of an activity localized at the anterior tip of the embryo. The mutant phenotypes of swallow swa and exuperantia (exu) share with weak bcd alleles the lack of anterior head structures. Cytoplasmic transplantations reveal a much reduced $\mathrm{bcd}^{+}$activity at the anterior tip of $s w a$ and $e x u$ embryos. In contrast to weak bcd alleles, removal of anterior cytoplasm has very little effect on the swa or exu phenotype. We suggest that in embryos mutant for exu or swa the bcd activity is not restricted to the anterior tip but extends toward more posterior positions. These conclusions are supported by changes in the fate maps of bcd, exu, and swa mutant embryos and by the ectopic expression of anterior structures in certain double-mutant phenotypes.
\end{abstract}

[Key Words: Drosophila; bicoid; antero-posterior axis; fate map; maternal genes]

Received June 30, 1987; revised version accepted August 12, 1987.

The segmented pattern of the Drosophila embryo, like that of other insects (Sander 1976; Kalthoff 1979), is organized by two terminal centers located at the anterior and the posterior egg poles. They are both defined by localized and transplantable cytoplasmic activities and by the phenotypes and properties of maternal-effect genes (Frohnhöfer et al. 1986; Frohnhöfer and Nüsslein-Volhard 1986; Lehmann and Nüsslein-Volhard 1986). The posterior pole plasm harbors factors for pole cell determination as well as an activity that organizes polarity and segmentation of the abdomen. These properties are dependent on the expression of several maternal genes including oskar, vasa, valois, staufen, and tudor (Boswell and Mahowald 1985; Lehmann and Nüsslein-Volhard 1986; Schüpbach and Wieschaus 1986; R. Lehmann and C. Nüsslein-Volhard, in prep.).

Whereas genes affecting the posterior center have very similar phenotypes regarding their effect on the posterior pattern, the phenotypes of genes affecting the anterior pattern show only a superficial resemblance to each other. Of these genes, the bicoid $(b c d)$ locus is unique with respect to its phenotype and organizing properties (Frohnhöfer and Nüsslein-Volhard 1986). In mutant bcd embryos, head and thorax are lacking and replaced by a duplicated posterior telson. $b c d^{+}$activity organizes the anterior pattern: Anterior cytoplasm from wild-type embryos transplanted into $b c d$ embryos restores a nearnormal pattern. When it is transplanted into more posterior positions, an anterior pattern (often accompanied by polarity reversals) can be induced at ectopic positions. $b c d^{+}$activity is localized in the anterior $15 \%$ of the egg (Frohnhöfer and Nüsslein-Volhard 1986) as is the bcd mRNA (Frigerio et al. 1986).
Two further groups of genes have been identified that affect anterior development. Mutations in these genes, however, remove only parts of the anterior head region. In this respect, they resemble weak $b c d$ alleles. Mutants of the torso group eliminate both terminal parts of the longitudinal pattern, posteriorly the telson and anteriorly the labrum (Degelmann et al. 1986; Schüpbach and Wieschaus 1986). Significantly, mutations in the genes of the posterior center and in $b c d$ do not eliminate development in the terminal regions, though in bcd mutant embryos, a telson develops anteriorly instead of the labral structures. Therefore, the torso group of genes appears to specify the terminal regions as a third entity of the longitudinal pattern. Depending on the presence of $b_{c d} d^{+}$activity, a labrum or a telson will arise from these terminal areas.

Embryos mutant for exuperantia (exu; Schüpbach and Wieschaus 1986) and swallow (swa; Gans et al. 1975; Mohler 1977; Stephenson and Mahowald 1987) have pattern defects in the head region that make them distinct from both the torso group genes and $b c d$. We have examined their effect on the $b c d$-dependent functions. We here present evidence that in exu and swa mutant embryos, the $b c d^{+}$activity is more evenly distributed than in wild-type embryos and suggest that both genes are involved in the anterior localization of $b c d^{+}$activity.

\section{Results}

The phenotypes in cuticular preparations: bicoid

Weak $b c d$ alleles cause reduction and deletion of structures in the anterior head region. With increasing allelic 
strength, all derivatives of the head and also the thorax are affected, whereas elements of the posterior telson are duplicated at the anterior. In addition, segmentation defects appear in the anterior abdomen. In strong alleles, head and thorax are lacking entirely.

A remarkable feature of the $b c d$ hypomorphic series is the lack of a strict antero-posterior order of segments deleted with increasing allelic strength. Although the anterior-most head structure, the labrum, is the structure most sensitive to a reduction of $b c d$ activity, the thoracic region may be already reduced in size when most of the other head elements are still present (although much smaller than normal). In addition to the anterior defects, most alleles show temperature-dependent segmentation defects in the abdomen. These consist of skipping or fusion of denticle bands, most frequently that of the abdominal segment 4 (A4) but also of A2 and A3. In severe cases the element of A2-A5 are involved, whereas the posterior-most abdominal segments $\mathrm{A} 6-\mathrm{A} 8$ remain normal. With few exceptions, the denticle band of $\mathrm{Al}$ is at least partially preserved in $b c d$ embryos. Its identity was confirmed by introducing $U b x$ into the progeny of $b c d$ females. In homozygous $U b x$ larvae, only the first abdominal segment is transformed into thorax (Lewis 1978; Struhl 1984). Figure 1D shows a Ubx/Ubx embryo derived from a $b c d^{E 1}$ female with a partially transformed anterior denticle band.

In the hypomorphic alleles $b c d^{E 3}$ (Fig. 1B), $b c d^{E 4}$, and $b c d^{111}$, there is a general correlation between the strength of the defects in head, thorax, and abdomen (though the abdominal defects depend inversely on temperature, see below). Two alleles, however, show peculiarities such that their position within the phenotypic series is ambiguous. $b c d^{E 5}$ causes only slight head defects (the labrum is frequently not eliminated), whereas comparatively strong segmentation defects occur in thorax and abdomen (Fig. 1C). Thus, small defects are spread over a large area (dispersed deletion profile). In contrast, in $b c d^{2-13}$ rather strong reductions of the anterior head region are not accompanied by any defects in the thorax or in the abdomen (localized deletion profile, Fig. 1A). In transheterozygous combinations, $b c d^{2-13}$ is enhanced more strongly by a deficiency than by any strong point mutation, whereas in combinations with other weak alleles, strong point mutations are indistinguishable from deficiencies.

exuperantia. The cuticular phenotype of embryos produced by exu females has been described by Schüpbach and Wieschaus (1986). Superficially, the exu phenotype resembles that of weak $b c d$ alleles in that anterior head structures are deleted (Fig. 1E). On closer inspection, however, it deviates from the weak $b c d$ phenotype. It has a very local deletion profile, and deletion of the anterior-most structures is never associated with defects in the thorax or anterior abdomen. In contrast, the remaining anterior regions appear enlarged rather than reduced in size: When only the labrum is eliminated $\left(29^{\circ} \mathrm{C}\right)$, the antennal sense organ still has its normal size; when both labrum and antenna are eliminated $\left(18^{\circ} \mathrm{C}\right)$, the maxillary structures are often increased in size. Moreover, the thoracic region in exu embryos is ex- panded as judged by its absolute length $289 \pm 8 \mu \mathrm{m}$, $n=15$, as compared to $220 \pm 8 \mu \mathrm{m}, n=10$, in newly hatched wild-type embryos) and an increased number of denticles in the prothorax.

Some duplication of posterior structures at the anterior tip is seen in exu embryos as well. This duplication rarely includes external cuticular structures but can be seen during gastrulation as a duplicated posterior midgut plate (Schüpbach and Wieschaus 1986). In contrast to $b c d$, occasional abdominal defects in exu are restricted to the posterior abdominal region. More frequently, segmentation is normal, but the overall size of the hind end is reduced (Fig. 1E).

swallow. swa and exu are fairly similar in phenotype, but in swa the head defects are somewhat weaker whereas the abdominal defects are stronger. At permissive conditions $\left(29^{\circ} \mathrm{C}\right)$ swa embryos are hardly distinguishable from exu in phenotype (Fig. 1F). However, at low temperature $\left(18^{\circ} \mathrm{C}\right)$ all $s w a$ embryos display abdominal segmentation defects, the majority retaining less than six denticle belts $\left(s w a^{14} / s w a^{-}\right)$(Fig. $\left.1 G, H\right)$. Furthermore, many embryos are already abnormal early during cleavage and fail to complete cellularization (Zalokar et al. 1975). Embryos may die before differentiation, and differentiated embryos frequently show defects all along the body. The head structures are completely absent occasionally, although this feature is likely to be caused by irregularities in cellularization rather than by a change in the patterning system.

For both swa and exu, the phenotypic variations with temperature (see below) are greater than interallelic differences. The two alleles of swa studied differ with respect to frequency and extent of abdominal defects; combinations with the deficiency indicate that neither allele is amorphic. The exu alleles are almost identical in strength. The exu phenotype was not clearly enhanced when the females were heterozygous for a synthetic deficiency constructed with aneuploids (see Experimental procedure) and a point mutant $\left(e x u^{P T}\right)$.

In summary, whereas all three genes affect the development of the anterior-most structures of the embryo, posterior structures are also affected, suggesting that the functions of these genes concern processes determining the pattern of a large region of the egg. The phenotypes of the genes exu and swa are quite similar to each other and distinct from that of bicoid.

\section{Temperature dependence}

The phenotypes of mutant alleles at all three loci are dependent on the temperature. In most hypomorphic bcd alleles, anterior defects are stronger when the embryos are raised at $29^{\circ} \mathrm{C}$ than at $18^{\circ} \mathrm{C}$, whereas the abdominal phenotype is cold sensitive. The most striking change of phenotypic expression with temperature is found in the allele $b c d^{E 3}$. At $29^{\circ} \mathrm{C}$, an almost amorphic phenotype is developed, whereas at $18^{\circ} \mathrm{C}$, the thorax is only slightly reduced and head structures are still prominent. Figure $2 \mathrm{~A}$ describes the temperature-sensitive period of $b c d^{E 3}$ as determined by shifting eggs collected at various times after egg deposition, between $18^{\circ} \mathrm{C}$ and 


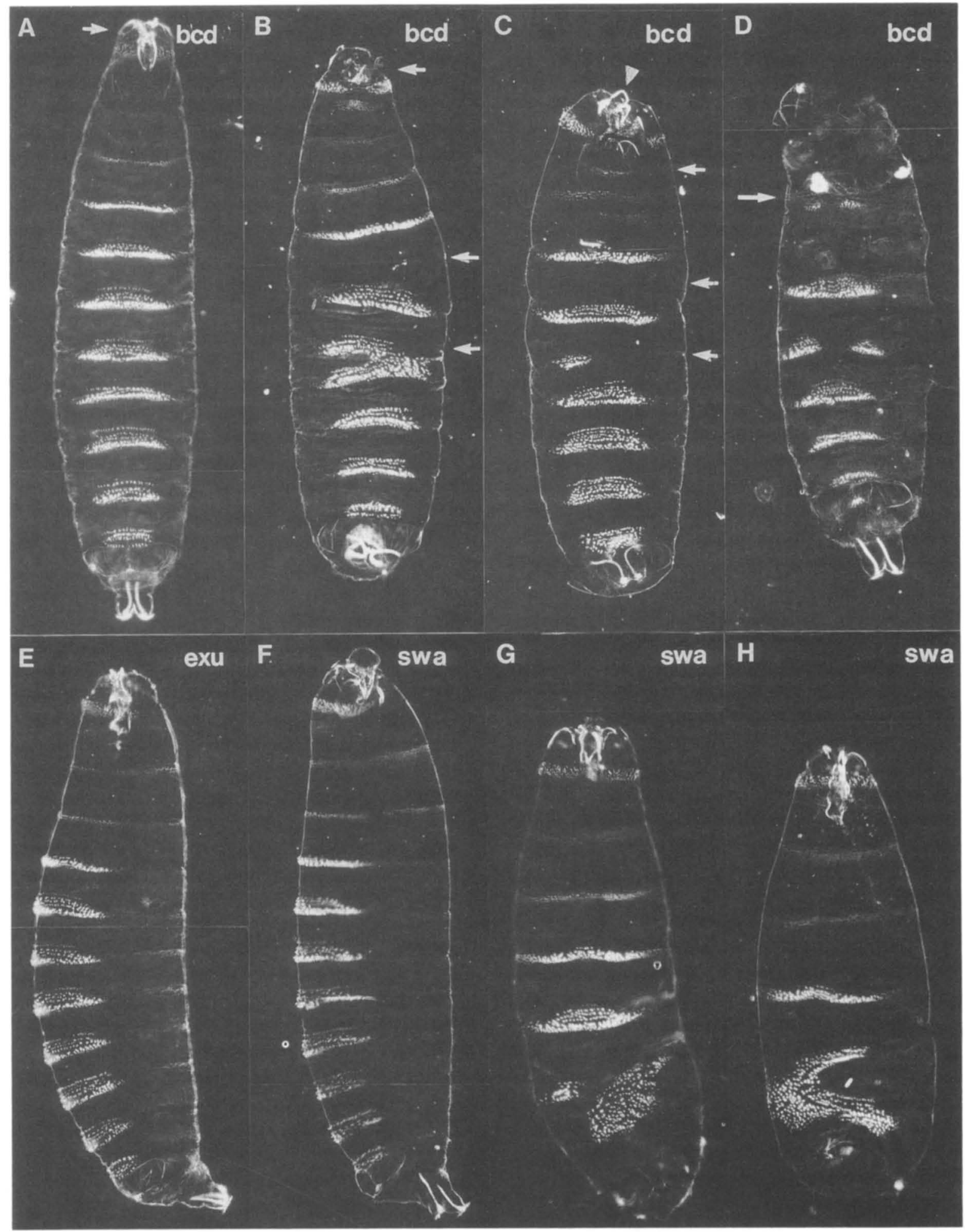

Figure 1. Cuticular patterns of $b c d$, exu, and $s w a$ mutant embryos. The genotypes of the females producing the embryos shown

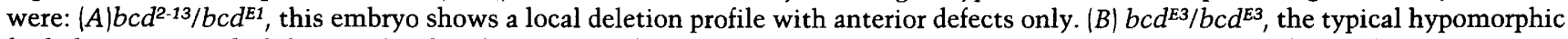
$b c d$ phenotype with defects in head and anterior abdomen (arrows). $(C) b c d^{E 3} / b c d^{E 5}$, dispersed deletion profile with strong pattern defects in thorax and anterior abdomen (arrows), whereas the head is rather complete as can be seen by the presence of a labrum (triangle). (D) $b c d^{E 1} / b c d^{E 1}$, the strong $b c d$ phenotype. Head and thorax are replaced by a duplicated telson, and the anterior abdomen is defective. The embryo is homozygous for $U b x^{109} / U b x^{79 f}$; its anterior-most denticle band seems to be a composite of denticles derived from both $\mathrm{A} 1$ and $\mathrm{A} 2$, the former of which are transformed by $U b x$ and show thoracic morphology $(\operatorname{arrow}) .(E) e x u^{P I / e x u^{P I}}$. $(F-H)$ $s w a^{14} / D F(1) / F 5$. All embryos show the $18^{\circ} \mathrm{C}$ phenotype, except for the exu and swa embryos in $E$ and $F\left(22^{\circ} \mathrm{C}\right)$. 

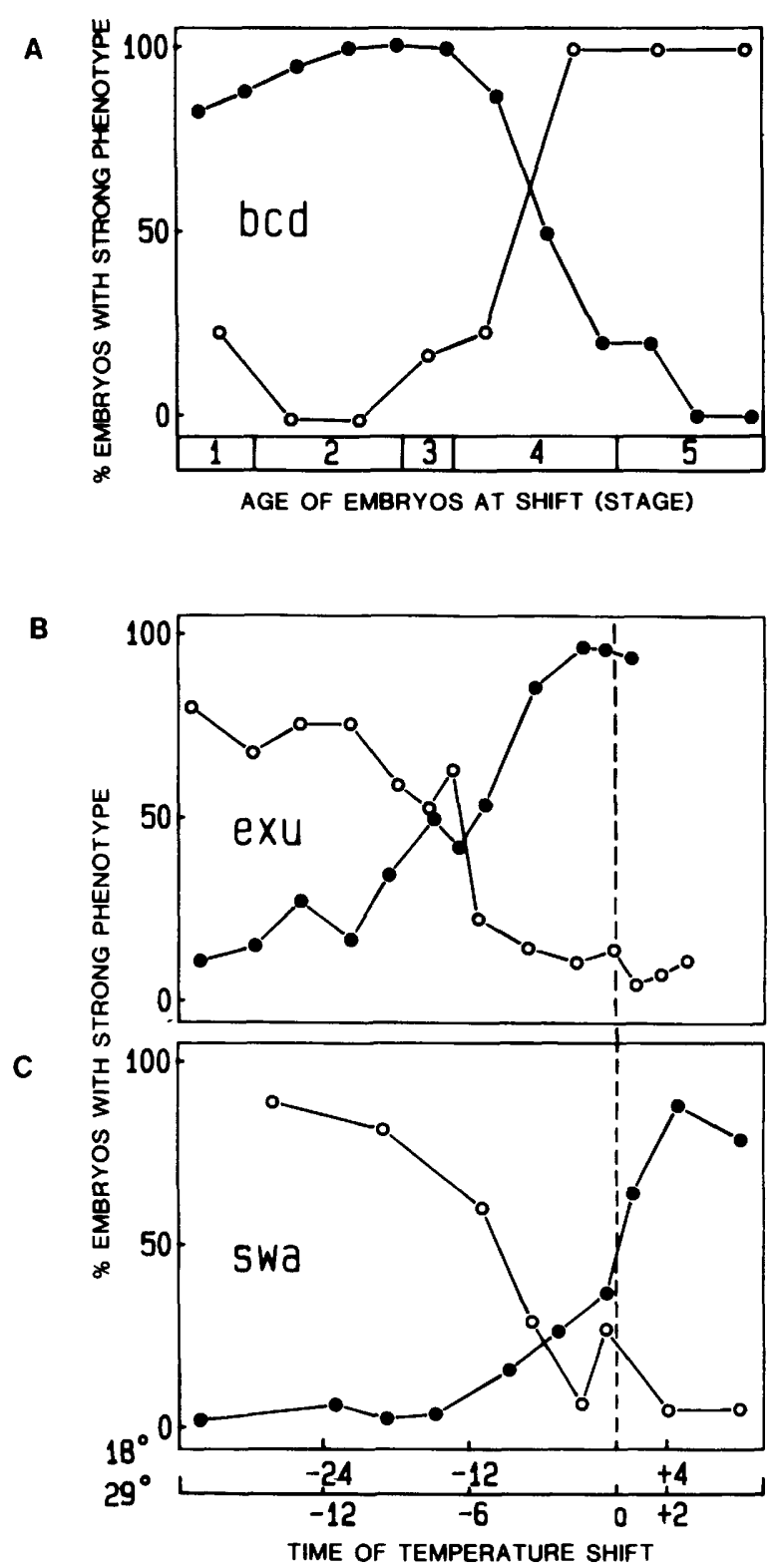

Figure 2. Temperature-sensitive periods of the phenotypes of bcd $(A)$, exu $(B)$, and swa $(C)$. The time scale in $A$ indicates the developmental times in stages defined by Campos-Ortega and Hartenstein (1985) [(1,2) intravitelline cleavage; (3) pole cell formation; (4) syncytial blastoderm; (5) cellularization of blastoderm]. The vertical dotted lines in $B$ and $C$ indicate the time of oviposition. (O) Shift down; (O) shift up. Each point represents at least 30 embryos.

$29^{\circ} \mathrm{C}$. The phenotypic strength recorded as defects both in the abdomen (not shown) and in the anterior pattern of embryos developing after such shifts reveals a phenocritical period that begins around pole cell formation (stage 3) and lasts until the formation of cell membranes between the blastoderm nuclei during stage 5 . This time coincides with the latest time at which phenotypic modifications can still be induced by transplantation of anterior cytoplasm (Frohnhöfer and Nüsslein-Volhard 1986), shortly before the first phenotypic manifestations be- come apparent during gastrulation. exu and $s w a$ are both cold sensitive. In exu, the anterior phenotype is enhanced, whereas in $s w a$, the abdominal defects are strongly increased at $18^{\circ} \mathrm{C}$. For both, the temperaturecritical period is during oogenesis (Fig. 2B,C). Temperature changes after egg deposition had little if any effect on the swa or exu phenotypes. As cold sensitivity is observed in all alleles and allele combinations tested, it is possible that the temperature effect reflects a property of the process affected rather than the feature of a particular mutant gene product.

\section{Fate maps}

Observation of living mutant embryos during gastrulation reveals that the basic phenotypic features as observed in the differentiated embryos take their origin from an early alteration of cell fate in the embryos (Frohnhöfer and Nüsslein-Volhard 1986; Schüpbach and Wieschaus 1986). To study the early fate maps, we determined the expression pattern of the pair rule gene fushi tarazu ( $f \mathrm{tz}$ ) in mutant embryos. We used a transformant line involving a construct linking the $f t z$ promoter to the $\beta$-galactosidase gene from $E$. coli (Hiromi et al. 1985) as well as a polyclonal antibody against a $f t z-\beta$-galactosidase ( $\beta$-gal) fusion protein (Carroll and Scott 1985). Both techniques yielded similar results. As the expression pattern of $f t z$ can be seen earlier with the antibody than by staining for $\beta$-gal activity, the former data were used to construct fate maps for mutant embryos.

In the cellular blastoderm stage of wild-type embryos, the $f t z$ gene is expressed in seven regularly spaced stripes positioned around the segmental boundaries of every other segment, from A8/9 at about $15 \%$ EL to the border between the labial and the maxillary segment located at about $65 \%$ EL just posterior to the cephalic furrow (Hafen et al. 1983; Carroll and Scott 1985; MartinezArias and Lawrence 1985). Whereas the posterior-most stripe is five cells wide in the blastoderm, all other stripes and interstripes extend to about three cell diameters in the antero-posterior axis; the $f t z$ expression pattern at this early stage coincides approximately with the anlagen of the even-numbered parasegments PS2-PS14 (Figs. 3A, 4; Martinez-Arias and Lawrence 1985; Ingham and Martinez-Arias 1986).

In $b c d$ embryos, the stripes are shifted to the anterior. This effect is observed even in embryos from $b c d$ heterozygous females. With increasing allelic strength, the expansion of the $f t z$ pattern anteriorly is enhanced (Figs. 3 and 4). The anterior two stripes (PS2 and PS4) become weak and finally disappear, whereas both the third stripe (marking the boundary between thorax and abdomen) and its distance to the fourth stripe is considerably enlarged (Fig. 3B,C, allele $b c d^{E 4}, b c d^{E 5}$ ). It is noteworthy that the allele-specific features observed in the cuticular phenotypes are also expressed in the stripe pattern: With a moderate anterior shift of the entire pattern, the PS6stripe is already considerably enlarged in $b c d^{E 5}$ embryos, whereas in $b c d^{2-13}$ embryos the anterior shift is more pronounced and affects all stripes in a regular manner 
(Fig. 3E). In strong alleles, a new stripe appears anteriorly that corresponds to a duplicated posterior stripe (PS14; Fig. 3D). In older embryos (containing the $f t z-\beta$-gal con- struct and stained for $\beta$-gal activityl, it borders the opening of the duplicated proctodeal invagination, which confirms its identity as a PS14-like stripe. Apart
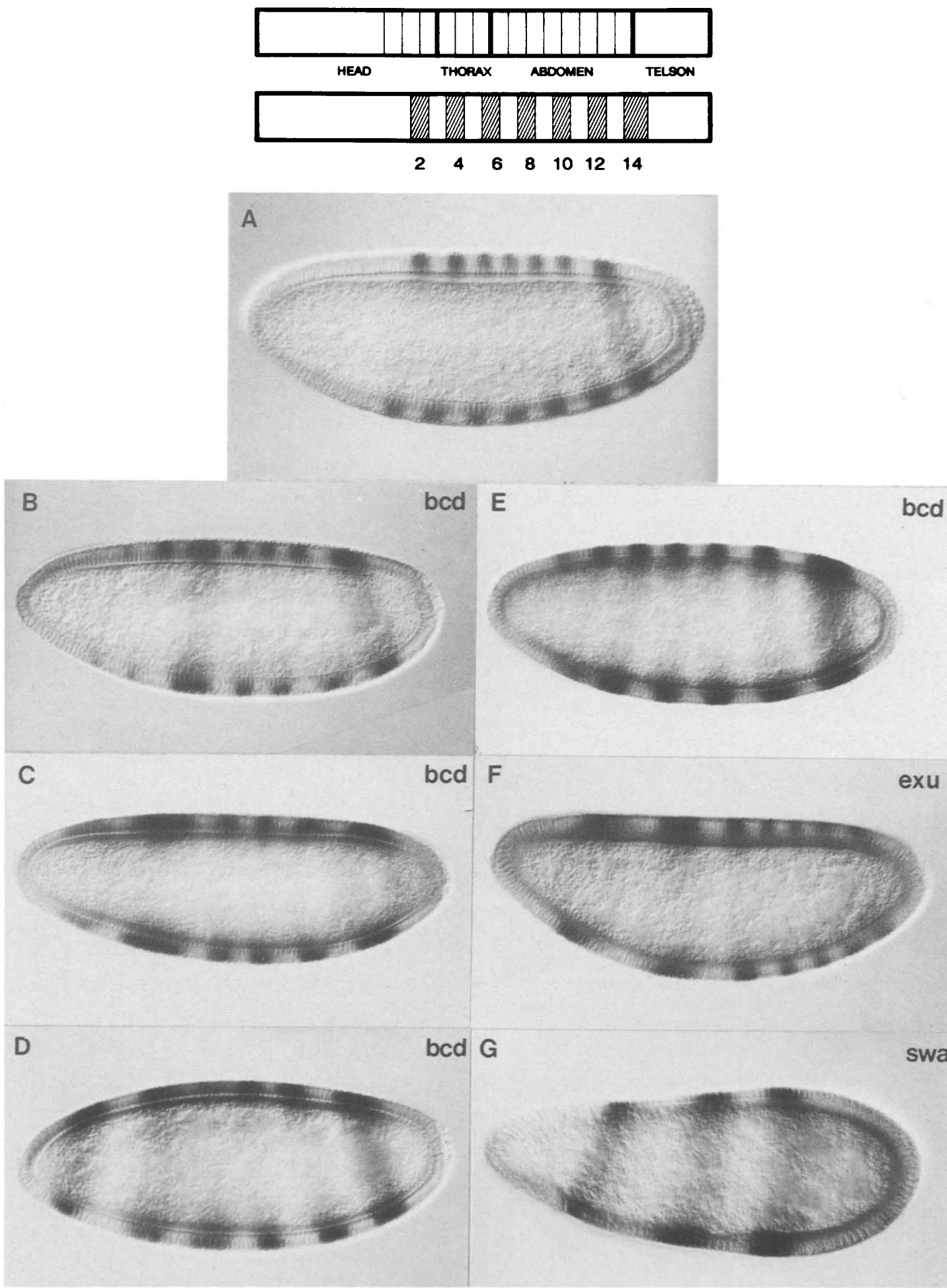

Figure 3. Expression pattern of $f t z$ in mutant embryos. An antibody against a $f t z-\beta$-gal fusion protein was bound to embryos from females of the genotypes wild type $(A), b c d^{E 5} / D f(3 R) L I N(B), b c d^{E 4} / D f(3 R) L I N(C), b c d^{33-5} / D f(3 R) L I N(D), b c d^{2-13} / D f(3 R) L I N(E), e x u^{P I /}$ exu $u^{Q R}(F)$, and $s w a^{14} / D f(1) J F 5(G)$, at $18^{\circ} \mathrm{C}$ and its distribution pattern revealed by processing with the biotin-avidin horseradish peroxidase staining system. The upper two panels indicate the positions of the parasegmental anlagen $2-14$ and the segments of head, thorax, and abdomen in relation to the wild-type staining pattern. 
from this, the total number of stripes in strong alleles never exceeds five, and by fusion of the anterior two stripes (PS6 and PS8) it may even be reduced to four (at lower temperature).

The final bcd phenotype thus reflects two distinct changes in the fate map: a reduction of anterior and an expansion of posterior anlagen. The reduction and deletion of gnathal and thoracic segments corresponds to a weakening and eventual disappearance of the anterior two $f t z$ stripes. In the abdomen, no primordia seem to be eliminated, but the segmentation is distorted. Whereas the abdominal anlage is expanding, its anterior region fails to become separated into distinct realms of $\mathrm{ftz}$ expression, but no particular stripe is deleted.

The change of the $f t z$ expression pattern in exu embryos is quite different from that in bcd embryos. Here it is the posterior anlagen that are compressed and crowded whereas the anterior two stripes are much enlarged and further apart than normal. No loss of stripes is observed in exu embryos, but the anterior stripe is shifted far toward the anterior pole, reaching $80 \% \mathrm{EL}$ (compared to $65 \%$ in the wild-type) (Fig. 3F, 4). Anterior expansion and posterior crowding appear as almost discontinuous and distinct alterations of the fate map: the reduced distance and size of the four posterior stripes is in sharp contrast to the more than doubled size and distance between the anterior two stripes. It is likely that the posterior segmentation defects occasionally observed in exu embryos are caused by the crowded parasegmental anlagen, whereas the substantial enlargement of the anterior anlagen results in the oversized gnathal and thoracic segments. In their study of $f t z$ expression in
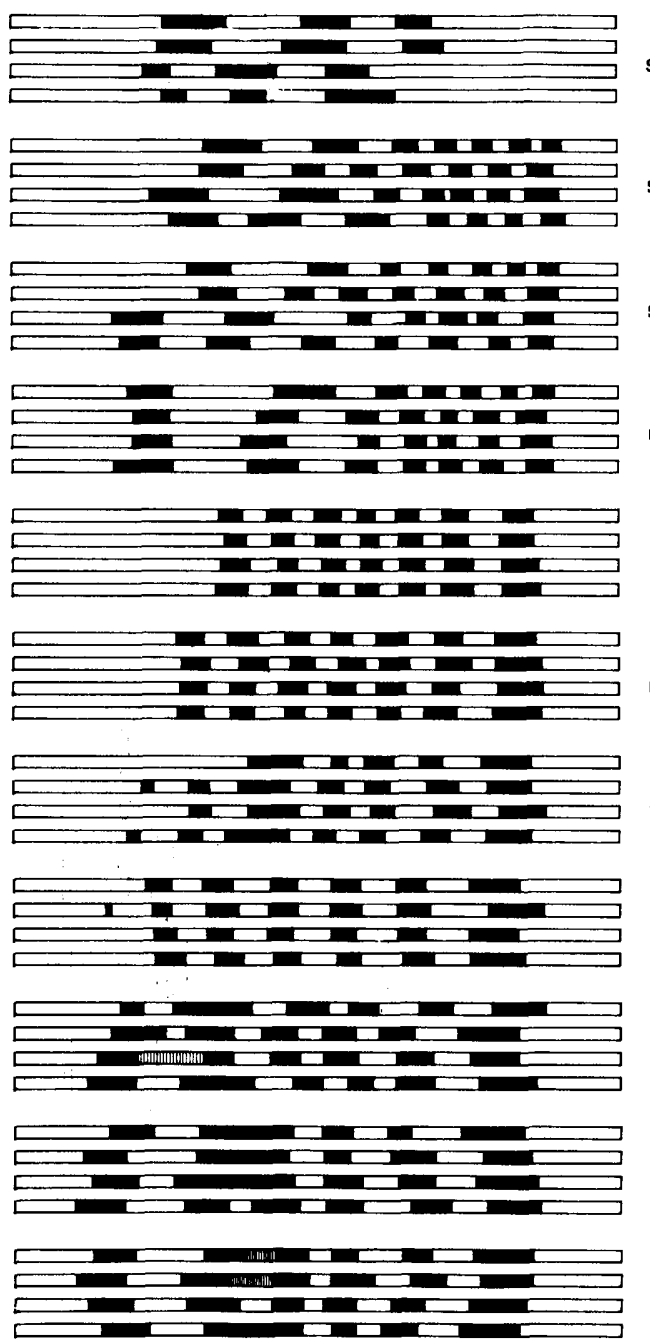

33-8

bed $/ D f$

E2 bcd $/ 0 f$

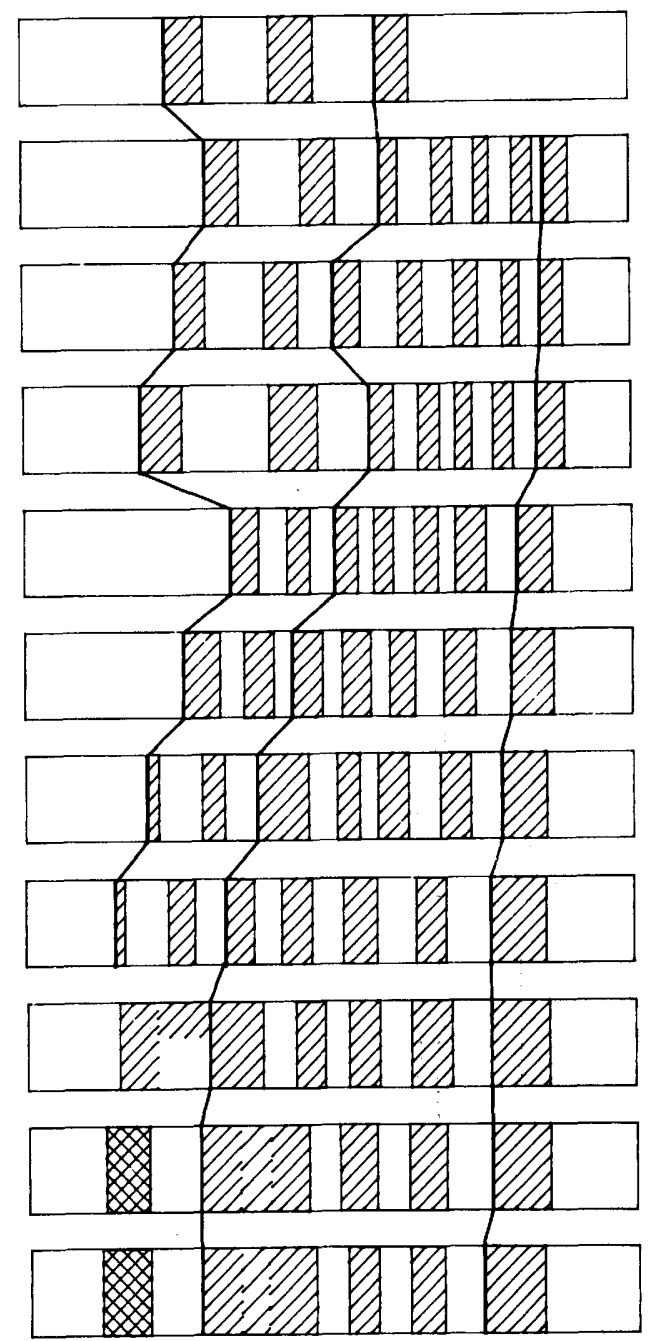

A

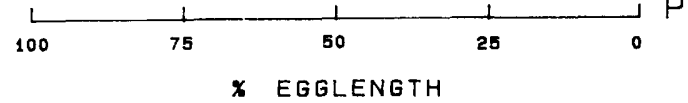

Figure 4. Patterns of $f t z$ expression in mutant and wild-type embryos. (Left) Each line represents one embryo, and for every genotype four embryos are represented. The maternal genotypes are indicated at the right. If not otherwise stated, eggs were collected at $18^{\circ} \mathrm{C}$. (Right) Average of the data presented. The anterior margins of the bands corresponding to parasegments, 2,6, and 14 are connected by heavy lines to indicate the relative shift of head, thorax, and abdomen. For further explanation see text. 
mutants affecting the posterior center, Carroll et al. (1986) have also observed that compression of the anlagen results in pattern defects, whereas expansion is tolerated with very little effects on the final pattern.

Although following the same tendency, the patterns of $f t z$ expression in $s w a$ embryos are more variable than those in exu. In weak alleles and generally at elevated temperatures the stripes appear expanded at the anterior and crowded at the posterior (Fig. 4). The transition between crowding and expansion is more continuous than the rather abrupt change in exu embryos. At low temperature, many embryos do not reveal a $f t z$ expression pattern due to lack of normal cellularization, whereas the stripe pattern in the developing embryos is often quite irregular. The anterior three stripes persist but their orientation with respect to the dorso-ventral or right-left axis is often oblique. The posterior stripes are weaker and less sharply defined, and frequently they disappear altogether (Fig. 3G).

\section{Reduction of anterior $\mathrm{bcd}^{+}$activity in exu and swa embryos}

To test whether the head defects observed in exu and $s w a$ embryos can be related to the $b c d^{+}$activity at the anterior pole, anterior cytoplasm from exu and swa was transplanted into bcd embryos. Whereas cytoplasm taken from the anterior tip of unfertilized wild-type embryos induces at least thoracic but frequently also head structures, the cytoplasm from exu and swa embryos is much less efficient (Fig. 5). None of the injected embryos was able to differentiate head structures. The reduction in $b c d^{+}$activity appears to be stronger in exu than in $s w a$, in agreement with the more pronounced effect of exu on anterior head development; indeed, in exu embryos a $b c d$-rescuing activity is barely detectable.

\section{Removal of anterior cytoplasm}

Leakage of anterior cytoplasm from wild-type eggs creates embryos with reduced head structures, similar to weak $b c d$ phenotypes. When cytoplasm is removed from embryos with an already reduced amount of $b c d^{+}$activity, phenocopies of $b c d$ with all phenotypic features including the abdominal segmentation defects result (Frohnhöfer and Nüsslein-Volhard 1986). These experiments suggest that the $b c d$ phenotype is caused entirely by the reduction or lack of anteriorly localized cytoplasmic activity. When cytoplasm is removed from the anterior tip of exu or swa embryos, a different result is obtained. Rather than increasing in phenotypic strength, the phenotypes remain more or less unmodified (Fig. 6). Thus, a reduction of $b c d^{+}$activity in eggs of weak $b c d$ alleles confers an increased sensitivity to loss of anterior cytoplasm, whereas in exu and swa embryos a reduced activity at the anterior pole is associated with an increased resistance against this treatment. This finding suggests that $b c d^{+}$activity in exu and swa embryos is not restricted to the anterior tip but that it is distributed toward more posterior positions in the embryo.

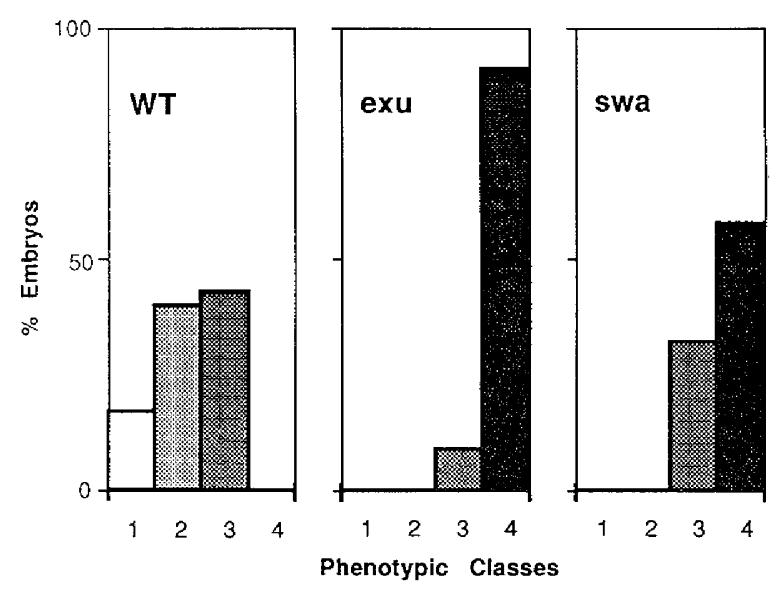

Figure 5. $b c d^{+}$activity at the anterior of exu and $s w a$ embryos. $b c d$ embryos were injected with cytoplasm taken from the anterior of wild type exu $u^{P I / e x u^{Q R}}$ or $s w a^{14} / s w a^{15}$ eggs as indicated. The resulting phenotypes were classified according to the degree of anterior development: (class 0) normal (appearing in Fig. 6 only); (class 1) development of thorax and a substantial portion of the head including antennal sense organs; (class 2) thorax and head structures but no antennae developed; (class 3 ) thorax but no head structures; (class 4) unmodified bicoid phenotype as observed in uninjected control embryos.

\section{Double mutant phenotypes}

Though exu and swa embryos are not responsive to loss of anterior cytoplasm, their phenotypes are strongly enhanced when $b c d^{+}$is reduced by genetic means. In embryos from $b c d^{-} /+$, exu/exu females, both head structures and prothorax are lacking; in combinations of exu with homozygous weak $b c d$ alleles, further thoracic segments are deleted in a strictly polar order, leaving a somewhat enlarged but otherwise normally segmented abdomen (Fig. 7B). Thus, exu confers a localized deletion mode to all levels of the $b c d$ hypomorphic series. However, in combinations with strong alleles $(e x u / e x u ; b c d /$ $b c d$, exu no longer influences the phenotype (Fig. 7A), supporting the notion that all phenotypic features of exu are mediated by its effect on $b c d^{+}$.

swa exhibits similar but less pronounced effects in combinations with $b c d$ alleles. The abdominal defects of swa seem to be ameliorated by reduced levels of $b c d^{+}$ activity, in accordance with the view that wrongly localized $b c d^{+}$activity is responsible for them. In contrast, the early cellularization defects are not suppressed by this means. In strong double mutant combinations (swa/swa; $b c d / b c d, 18^{\circ} \mathrm{C}$ ) a novel phenotype, bicaudal embryos, is frequently observed $130-60 \%$ of all developed embryos). The facilitation of bipolar development by swa mutant conditions suggests that swa, in contrast to exu, does not act solely on $b c d$ but is also involved in other functional circuits, which may not be immediately related to pattern formation, however.

Double mutants of exu and swa show an enhanced frequency of strong pattern defects; most embryos show the strong swa phenotype with rather distorted development in both the anterior and posterior parts of the em- 

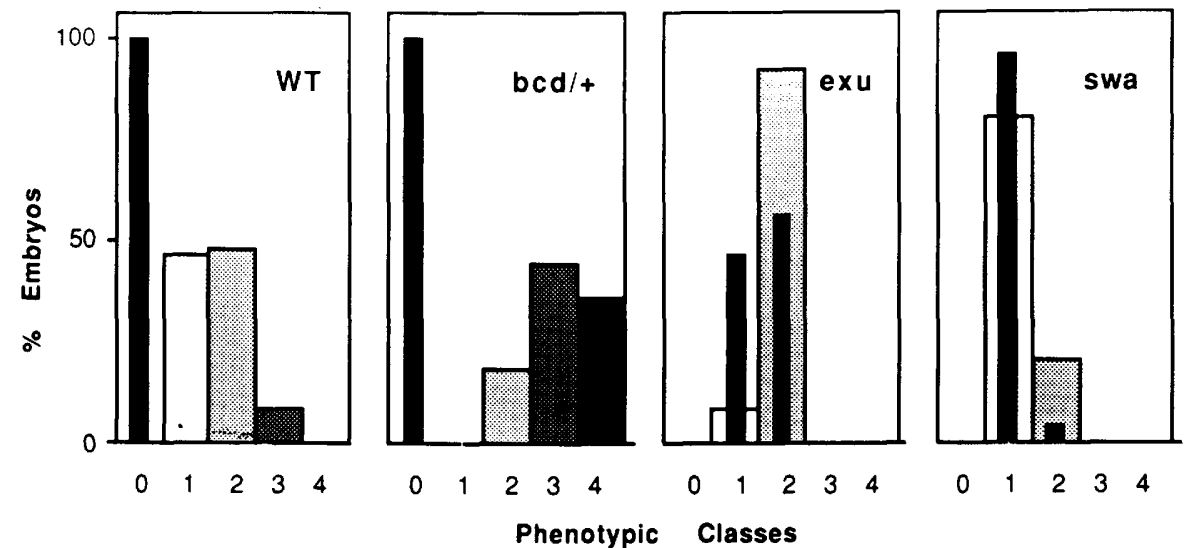

Figure 6. Modification of anterior development by removal of cytoplasm. Embryos from wild type, $D f(3 R) 9 A 99 /+, e x u^{P /} / e x u^{Q R}$, and $s w a^{14} / s w a^{15}$, respectively, were pricked at the anterior tip in early cleavage and the leaking cytoplasm removed. The resulting phenotypes were classified as in Fig. 5. The narrow black bars indicate the phenotypic frequencies of unpricked control embryos.

bryo. However, no entirely new phenotype is created, nor do the effects of exu and swa add up to a strong bcd phenotype.

In addition to the anterior $b c d$ activity, the activity localized in the posterior pole plasm (posterior center) confers polarity to the early embryo (Lehmann and Nüsslein-Volhard 1986). The elimination of the posterior polarizing center in exu embryos results in mirrorimage duplications of anterior structures: vasa exu double mutant females produce embryos with duplicated gnathal regions whereas thorax, abdomen, and telson structures are lacking (Schüpbach and Wieschaus
1986). Similar duplications can be found in the combination exu;oskar (osk): Mouthhooks or only prothoraces may be formed at both ends of the embryo. There are always only a few cuticular structures expressed in these animals. Frequently they do not differentiate any cuticular markers, possibly because little gradation of positional values is left (Fig. 7).

The combinations suggest that it is mainly the posterior pole plasm that accounts for polarity in exu single mutants, whereas almost no polarity is conferred by the bcd distribution on its own. Because the pole plasm is able to suppress the anterior $b c d^{+}$activity (Frohnhöfer

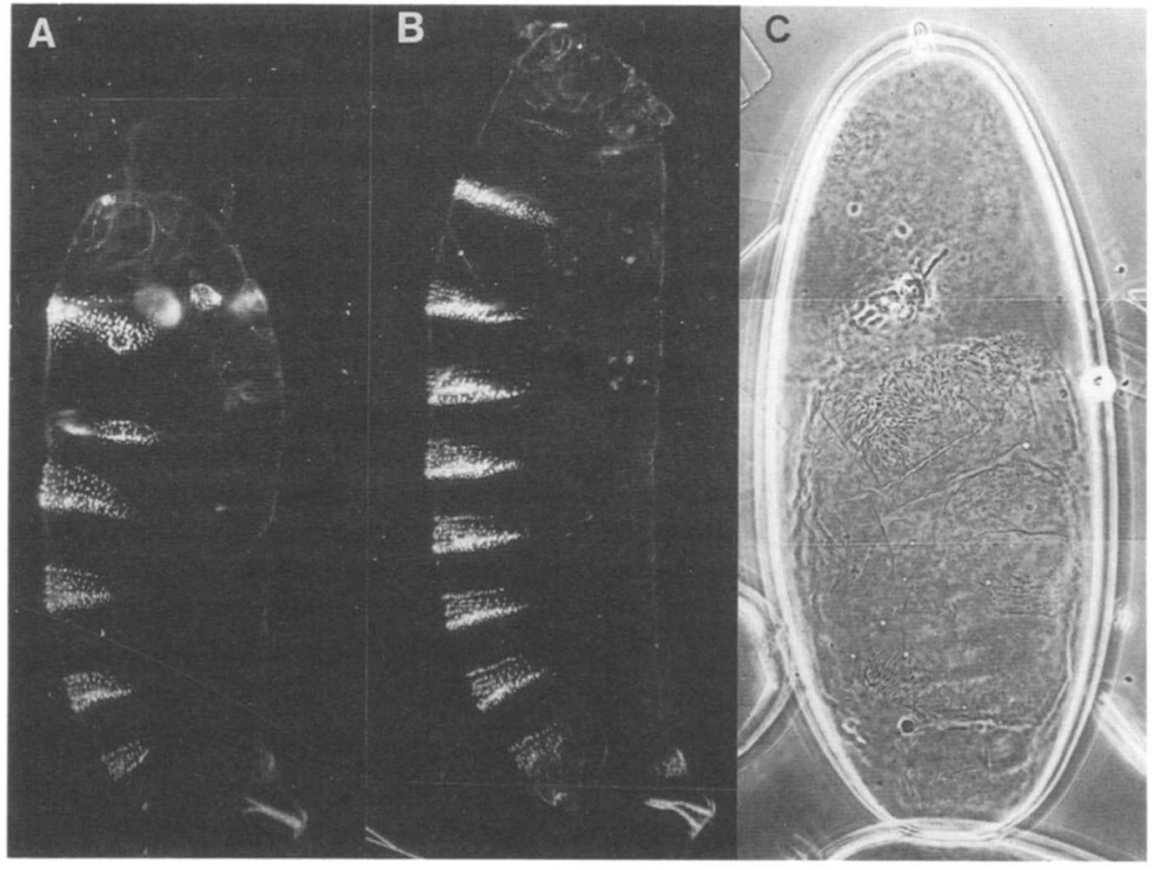

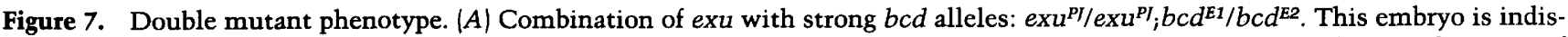
tinguishable from a strong $b c d$ embryo. $(B)$ Combination of exu with weak bcd alleles: $e x u^{P I} / e x u^{P I} ; b c d^{E 5} / b c d^{E 4}$. $(C)$ Combination of $e x u$ with osk:exu ${ }^{P I} / e^{P u^{P I}} ; o s k^{166 / o s k^{166}}$. The embryo displays prothoracic denticle bands at both ends. 
et al. 1986; Lehmann and Nüsslein-Volhard 1986), it can convert the symmetric state of exu;osk embryos toward the polar exu phenotype.

In $s w a$, more of the anterior polarity seems to be maintained, as swa;osk combinations do not assume a symmetric state. Rather, defects are superimposed on each other in the cuticle phenotype (whereas in the anlagen plan a posterior shift of the anterior structures would be predicted).

\section{Discussion}

Mutations in exu and swa affect both the anterior and posterior domain of the longitudinal pattern. Therefore, possible functions of these genes would be to affect the establishment of both polar centers or influence their interactions; alternatively, they might act on $b c d$ alone, which, in turn, mediates the abdominal defects. Our data support the latter interpretation. Specifically, they indicate that exu and swa are required for the localization of the bcd product at the anterior pole. In exu and swa mutant embryos, the posterior activity is not affected (data not shown), whereas the $b c d$ activity at the anterior pole is severely reduced. However, comparison with weak $b c d$ alleles shows that there is no simple reduction in anterior activity. Removal of cytoplasm from the anterior tip has almost no effect on the phenotype of $e x u$ and $s w a$, whereas hypomorphic alleles of $b c d$ react to this treatment with a strong enhancement of the phenotype. Because double mutants suggest that exu and $s w a$ are sensitive to reduction of $b c d^{+}$, resistance to loss of anterior cytoplasm suggests that in exu and swa the $b c d^{+}$activity has lost its normal, predominantly anterior localization and is present in more posterior positions.

Changes in the fate map demonstrate how the absence or mislocalization of $b c d^{+}$affects the general pattern. It has been shown before that $b c d^{+}$has some suppressive effect on abdominal development; in particular, if transplanted toward posterior positions it may cause the entire abdomen to disappear. In $b c d$ mutant embryos, the abdominal anlagen are spread anteriorly, obviously due to the lack of anterior restrictive influence. Thus, a more homogeneous distribution of $b c d^{+}$can account for the posterior pattern defects in exu and swa. Whereas anteriorly the $b c d^{+}$activity is no longer sufficient to induce the anterior-most structures of the head, its presence in more posterior positions pushes the posterior anlagen backwards. The spreading of intermediate levels of $b c d^{+}$ activity causes enlarged regions to become thorax and gnathocephalon.

It is difficult to measure $b c d^{+}$activity in medial and posterior egg positions by transplantation tests, because these regions also contain abdomen-specific factors that complicate the analysis by antagonistic interactions with $b c d^{+}$activity. Positive evidence for ectopic positioning of $b c d^{+}$activity in exu embryos rests mainly on the double mutants, with vasa and osk giving rise to phenotypes with a dicephalic symmetry. When the pole plasm in exu embryos is removed, almost no polarity is maintained, suggesting a fairly homogeneous distribution of $b c d^{+}$activity. In $s w a$, the $b c d^{+}$distribution may be less even. However, we do not know whether this difference is allele or locus specific.

All mutant effects of exu may be mediated by an even distribution of $b c d^{+}$activity, whereas swa appears to have additional functions. The reduced capacity to produce pole cells observed in swa embryos /data not shown/ may result from a reduced rate of early cleavage (Okada 1986); further, the frequent failure of proper cellularization points to a weakness in more general cellular physiology. Whereas in double mutants with $b c d$ the effects of exu are all eliminated or suppressed, the cellularization defects of swa cannot be suppressed by the elimination of the bcd product. swa also deviates from exu with respect to pattern formation. The two mutants might differ in the degree of $b c d$ homogeneity as well as in the total quantity of $b c d$-dependent product present in the egg. Transplantation experiments using exu and $s w a$ as source for $b c d^{+}$activity and interpreting the double mutants with osk suggest that in $s w a$, residual polarity is preserved and more $b c d^{+}$activity is present at least at the anterior pole, although these differences might be allele rather than locus specific. However, the weak effect on the abdomen compared with that on the head is a gene-specific peculiarity of exu that can be attributed to a lowered average amount of $b c d^{+}$ activity.

bcd mRNA is transcribed during oogenesis by the nurse cells and accumulates at the anterior end of the oocyte (Frigerio et al. 1986). As the oocyte grows, the RNA remains essentially in position and thus ends up at the anterior pole of the egg. No elaborate transport systems in the oocyte have to be assumed; the RNA could simply be trapped upon entry into the egg. At the anterior pole of young eggs, inhomogeneities can be seen in the yolk/cytoplasm distribution, but no cytoskeletal specializations have been described. Judging from our work, it seems that exu is more directly associated with the $b c d$ product, fixing it and possibly giving it some protection, whereas swa may be part of the general cellular framework facilitating localization. This could explain inversion of abdominal segments in double mutants of swa and bcd or swa and hunchback (not shown). However, such instabilities occurring with incomplete penetrance in both combinations may allow other interpretations.

It has been suggested that $b c d$ RNA acts as a source to produce a $b c d$ protein gradient, which specifies spatially restricted transcription of zygotic segmentation genes of the gap class and thereby organizes the anterior pattern (Frohnhöfer and Nüsslein-Volhard 1986). Assuming a shallow distribution of bcd RNA in exu embryos, the expansion of the gnathal and thoracic region in the fate map is the most direct evidence for a concentration-dependent specification of the pattern along the anteroposterior axis.

\section{Materials and methods}

Mutant strains

The bicoid alleles and $D F(3 R) L I N, b c d^{-}$have been described (Frohnhöfer and Nüsslein-Volhard 1986). The exu alleles were 
obtained from T. Schüpbach (Schüpbach and Wieschaus 1986). Since no chromosomal deficiency is available for exu, the cytological localization was determined by constructing synthetic deficiencies using $T\left(Y_{i} R\right.$ ) translocations (Lindsley and Sandler et al. 1972). Females of the genotype C(1)RM; T(Y:2R)L139P/ $T\left(Y_{i} 2 R\right) L 107^{D} / e x u^{P I}$, although poorly viable, produced some embryos with a strong exu phenotype placing exu into the cytological interval 56E;57B. The swallow alleles $s w a^{14}$ and $s w a^{15}$ are $f_{s}(1) 1497$ and $f_{s}(1) 1502$ (Gans et al. 1975). The swa deficiency, $D f(1) J F 5$, was kindly supplied by E. Stephenson and is described in Stephenson and Mahowald (1987). The wild-type stock was Oregon R. All mutant chromosomes contained suitable visible markers. Flies were grown under standard conditions (Nüsslein-Volhard et al. 1984), and eggs were collected and processed for the determination of the cuticular phenotypes as described (Wieschaus and Nüsslein-Volhard 1986).

\section{Temperature shift experiments}

The temperature-sensitive period of the allele $b c d^{E 3}$ was determined. Eggs were collected from $b c d^{E 3}$ homozygous females on yeasted agar plates for $3 \mathrm{hr}$ at $29^{\circ} \mathrm{C}$ or $7 \mathrm{hr}$ at $18^{\circ} \mathrm{C}$ and shifted to the other temperature at the end of the egg collection period. The eggs were covered with Voltalef $3 S$ oil and visibly staged by selecting gastrulating embryos (stage 6; Campos-Ortega and Hartenstein 1985) at regular intervals after the shift $(20 \mathrm{~min}$ at $29^{\circ} \mathrm{C}, 1 \mathrm{hr}$ at $18^{\circ} \mathrm{C}$. The batches of embryos were kept to develop at the final temperature. The embryos were fixed and mounted. The phenotype was scored as strong if the embryos lacked most head structures and had less than two thoracic segments. The cold-sensitive period of exu and swa lies before egg deposition. Females of the genotype $e x u^{P I / e x u^{P I}}$ or $s w a^{14 /}$ Df(1)JF5 were shifted from $18^{\circ} \mathrm{C}$ to $29^{\circ} \mathrm{C}$ and vice versa. Eggs were collected at regular intervals after the shift and the phenotypes determined. For $e x u$, the phenotype was graded as strong if the dorsal arms and the ventral plate of the cephalopharyngeal skeleton (Jürgens et al. 1986) was absent. The swa embryos were scored for incidence of developmental failure and segmentation defects in the posterior abdomen. They were scored as strong mutants when they had less than seven abdominal segments. Although the temperature-critical period using either criteria covers about the same developmental time, data concerning abdominal segmentation yielded the clearest results and are presented here. The frequency relates to the total number of embryos developing a scorable cuticular pattern.

\section{Antibody staining}

Embryos were dechorionated, fixed, and devitellinized with the heptane-methanol standard procedure (Mitchinson and Sedat 1983). The fixation was performed in $4 \%$ fresh paraformaldehyde in $0.1 \mathrm{M}$ PIPES (pH 6.9), $2 \mathrm{mM} \mathrm{MgSO}_{4}, 1 \mathrm{~mm}$ EGTA, and the devitellinization was carried out at room temperature rather than applying dry ice-hot water treatment. The first antibody, a polyclonal rabbit $a-f t z$, was kindly obtained from S.B. Carroll and M.P. Scott (Carroll and Scott 1985). Antibody binding and detection using horseradish peroxidase staining was according to the protocol of MacDonald and Struhl (1986). Embryos were dehydratd with ethanol, mounted in $50 \%$ dried Canada balsam dissolved in methyl salicylate, and hardened by overnight incubation at $60^{\circ} \mathrm{C}$. The positions of the strips were determined from camera lucida drawings of optical sections using Nomarski interference contrast. The mean lateral position of the stripes along the longitudinal egg axis was calculated using standard geometrical procedures. The four embryos of each genotype, the patterns of which are shown in Fig. 4a, were selected according to their age (middle stage 5) and suitable orientation and no corrections were applied. For several genotypes, a larger sample of embryos was used with little, if any, effect on the mean values shown in Figure 4B, with the exception of swa which shows considerable variability.

\section{Cytoplasmic transplantation}

Transplantation of cytoplasm from the anterior tip of donor embryos into the anterior tip of stage $2 b c d$ recipient embryos was carried out as described (Frohnhöfer and Nüsslein-Volhard 1986). The recipient embryos were from $b c d^{E 1} / b c d^{E 2}$ females. Uninjected control embryos show exclusively the strong $b c d$ phenotype (class 4). The donor eggs were from $s w a^{14} / s w a^{15}$, $e x u^{P I / e x u^{Q R}}$, or from Oregon- $\mathrm{R}$ females kept at $18^{\circ} \mathrm{C}$. All donor eggs were unfertilized and used within $3 \mathrm{hr}\left(18^{\circ} \mathrm{C}\right)$ after egg deposition. Removal of cytoplasm from the anterior tip of embryos from females of various genotypes was performed as described (Frohnhöfer et al. 1986; Frohnhöfer and Nüsslein-Volhard 1986). The frequency of embryonic phenotypes was calculated for embryos surviving the experimental procedure and developing a scorable cuticular pattern, usually $50-80 \%$ of all treated embryos. Each point represents an average of at least 35 embryos.

\section{Acknowledgment}

We thank T. Schüpbach and E. Stephenson for mutant stocks, S. Carroll and M. Scott for a generous gift of $a$-ftz antibody, and W. Driever for help with the antibody staining. We thank our colleagues for their interest, discussion, and comments on the manuscript, R. Groemke-Lutz for the photographic prints, and D. Kaiser for preparing the manuscript. This work was supported in part by the Deutsche Forschungsgemeinschaft (Leibniz-Programm).

\section{References}

Boswell, R.E. and A.P. Mahowald. 1985. tudor, a gene required for assembly of the germ plasm in Drosophila melanogaster. Cell 43: 97-104.

Campos-Ortega, J.A. and V. Hartenstein. 1985. The embryonic development of Drosophila melanogaster, pp. 9-84. Springer Verlag, Heidelberg.

Carroll, S.B. and M.P. Scott. 1985. Localization of the fushi tarazu protein during Drosophila embryogenesis. Cell 43: 47-57.

Carroll S.B., G.M. Winslow, T. Schüpbach, and M.P. Scott. 1986. Maternal control of Drosophila segmentation gene expression. Nature 323: 278-280.

Degelmann, A., P.A. Hardy, N. Perrimon, and A.P. Mahowald. 1986. Developmental analysis of the torso-like phenotype in Drosophila produced by a maternal-effect locus. Dev. Biol. 115: 479-489.

Frigerio, D., M. Burri, D. Bopp, S. Baumgartner, and M. Noll. 1986. Structure of the segmentation gene paired and the Drosophila PRD gene set as part of a gene network. Cell 47: 735-746.

Frohnhöfer, H.G. and C. Nüsslein-Volhard. 1986. Organization of anterior pattern in the Drosophila embryo by the maternal gene bicoid. Nature 324: 120-125.

Frohnhöfer, H.G., R. Lehmann, and C. Nüsslein-Volhard. 1986. Manipulating the anteroposterior pattern of the Drosophila embryo. J. Embryol. Exp. Morph. (Suppl.) 97: 169-179.

Gans, M., C. Audit, and M. Masson. 1975. Isolation and charac- 
terization of sex linked female sterile mutants in Drosophila melanogaster. Genetics 81: 683-704.

Hafen, E., A. Kuroiwa, and W.J. Gehring. 1984. Spatial distribution of transcripts from the segmentation gene fushi tarazu during Drosophila development. Cell 37: 833-841.

Hiromi, Y., A. Kuroiwa, and W.J. Gehring. 1985. Control elements of the Drosophila segmentation gene fushi tarazu. Cell 43: 603-613.

Ingham, P.W. and A. Martinez-Arias. 1986. The correct activation of Antennapedia and bithorax complex genes requires the fushi tarazu gene. Nature 324: 592-597.

Jürgens, G., R. Lehmann, and C. Nüsslein-Volhard. 1986. Segmental organization of the head in the embryo of Drosophila melanogaster. Wilhelm Roux's Arch. Dev. Biol. 195: 359-377.

Kalthoff, K. 1979. Analysis of a morphogenetic determinant in an insect embryo. Symp. Soc. Dev. Biol. 37: 97-126.

Lehmann, R. and C. Nüsslein-Volhard. 1986. Abdominal segmentation, pole cell formation, and embryonic polarity require the localized activity of oskar, a maternal gene in Drosophila. Cell 47: 141-152.

Lewis, E.B. 1978. A gene complex controlling segmentation in Drosophila. Nature 276: 565-570.

Lindsley, D.L., L. Sandler, B.S. Baker, A.T.C. Carpenter, R.E. Denell, J.C. Hall, P.A. Jacobs, G.L. Miklos, B.K. Davis, R.C. Gethman, R.W. Hardy, A. Hessler, S.M. Miller, H. Nozawa, D.M. Parry, and M. Gould-Somero. 1972. Segmental aneuploidy and the genetic gross structure of the Drosophila genome. Genetics 71:157-184.

MacDonald, M. and G. Struhl. 1986. A molecular gradient in early Drosophila embryos and its role in specifying the body pattern. Nature 324: 537-545.

Martinez-Arias, A. and P.A. Lawrence. 1985. Parasegments and compartments in the Drosophila embryo. Nature 313: 639.

Mitchinson, T.J. and J.W. Sedat. 1983. Localization of antigenic determinants in whole Drosophila embryos. Dev. Biol. 99: $261-264$.

Mohler, J.D. 1977. Developmental genetics of the Drosophila egg. I. Identification of 50 sex-linked cistrons with maternal effects on embryonic development. Genetics 85: 259-272.

Nüsslein-Volhard, C., E. Wieschaus, and H. Kluding. 1984. Mutations affecting the pattern of the larval cuticle in Drosophila melanogaster. I. Zygotic loci on the second chromosome. Wilhelm Roux's Arch. Dev. Biol. 183: 267-282.

Okada, M. 1986. Cytoplasmic function segregating germ line in Drosophila embryogenesis. Zool. Science 3: 573-583.

Sander, K. 1976. Specification of the basic body pattern in insect embryogenesis. Adv. Insect Physiol. 12: 125-238.

Schüpbach, T. and E. Wieschaus. 1986. Maternal-effect mutations altering the antero-posterior pattern of the Drosophila embryo. Wilhelm Roux's Arch. Dev. Biol. 195: 302-317.

Stephenson, E. and A.P. Mahowald. 1987. Dev. Biol. (in press).

Struhl, G. 1984. Splitting of the bithorax complex of Drosophila. Nature 308: 454-457.

Wieschaus, E. and C. Nüsslein-Volhard. 1986. Looking at embryos. In Drosophila: A practical approach (ed. D. Roberts), pp. 199-228, IRL Press, Oxford, Washington DC.

Zalokar, M., C. Audit, and I. Erk. 1975. Developmental defects of female sterile mutants of Drosophila melanogaster. Dev. Biol. 47: 419-437. 


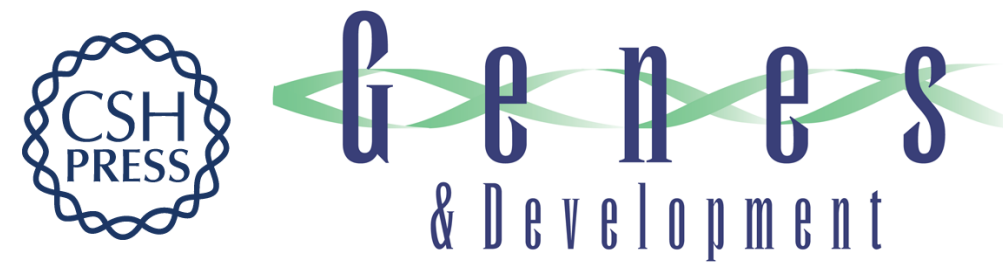

\section{Maternal genes required for the anterior localization of bicoid activity in the embryo of Drosophila}

Genes Dev. 1987, 1:

Access the most recent version at doi:10.1101/gad.1.8.880

References This article cites 26 articles, 3 of which can be accessed free at: http://genesdev.cshlp.org/content/1/8/880.full.html\#ref-list-1

License

Email Alerting

Receive free email alerts when new articles cite this article - sign up in the box at the top Service right corner of the article or click here.

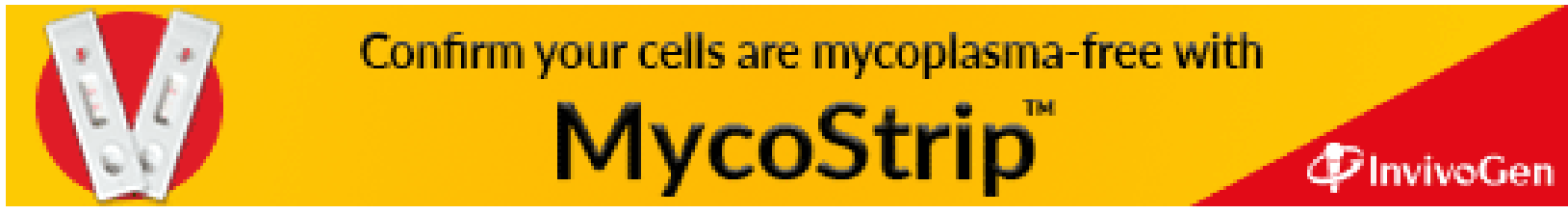

